\title{
A Single Session of Exercise Reduces Blood Pressure Reactivity to Stress: a Systematic Review With Meta-analysis
}

Igor M. Mariano

Federal University of Uberlândia

Ana Luiza Amaral

Federal University of Uberlândia

Paula A. B. Ribeiro

Research Center of University of Montreal Hospital Centre

Guilherme M.Puga ( $\sim$ gmpuga@ufu.br )

Federal University of Uberlândia

\section{Research Article}

Keywords: Aerobic Exercise, Resistance Exercise, Blood Pressure, Stress, Reactivity

Posted Date: September 20th, 2021

DOI: https://doi.org/10.21203/rs.3.rs-873062/v1

License: (c) (i) This work is licensed under a Creative Commons Attribution 4.0 International License. Read Full License 


\section{Abstract}

Stressful situations are common in everyday life and disturb homeostasis. So, an exercise session is a strategy to mitigate blood pressure (BP) peaks in response to stress (i.e., BP reactivity), decreasing the cardiovascular risk of these individuals. This is a systematic review with a meta-analysis that aims to verify the effects of a single session of physical exercises on BP reactivity to stress. The searches were realized in digital databases (PUBMED, LILACS, EMBASE and Psyclnfo) and 28 studies were included, totaling 846 individuals (meta-analysis stage: $k=24, n=710$ ). As for exercise characteristics, 23 of the 28 studies focused on aerobic exercises, and 24 studies focused on low to moderate intensities.

Favorable metanalytic results (standardized mean differences through random-effects approach) for the exercises were found, with attenuated reactivity in systolic BP (pooled effect size $=-0.35[-0.46 ;-0.23]$, representing average reductions of $3.8 \pm 3.5 \mathrm{mmHg}$ ), diastolic BP (pooled effect size $=-0.49[-0.68 ;-0.30]$, representing average reductions of $3.1 \pm 3.6 \mathrm{mmHg}$ ), and mean BP (pooled effect size $=-0.48$ [-0.70; $-0.26]$, representing average reductions of $4.1 \pm 3.0 \mathrm{mmHg}$ ). So, acute physical exercise lowers systolic, diastolic, and mean blood pressure reactivity in response to stressor tasks.

\section{Introduction}

Stressful situations are common in modern life and can cause alterations in autonomic, catecholaminergic and neural networks in response to it [1-3]. In this way, simple laboratory stress tests that disturb the homeostasis in a controlled manner was previously associated with the development of future cardiovascular events, depression and decreased telomere length [4]. This is accomplished through different types of stressors, such as physical (e.g., cold), mental (e.g., arithmetic task) or a mix of both [5]. To assess these responses, several markers are used [5], of which we will highlight the blood pressure (BP) alterations (i.e., hypertensive peaks).

In a broad context, high BP is one of the main preventable factors associated with premature death globally [6] and is associated with the risk of cardiovascular events, strokes and kidney disease [7]. In this context, one of BP's control strategies is to perform physical exercises. Evidence shows that even after a single exercise section, BP can be below baseline levels at rest [8] but its influence on BP reactivity to stressful situations is still poorly understood. Despite that, it has already been suggested that cardiovascular responses to stress are better indicators of left ventricular mass [9] and the development of hypertension $[10,11]$ then resting $B P$, reiterating the importance of studying these responses.

In 2006, a meta-analysis by Hamer and collaborators [12] evaluated the acute effects of aerobic exercise on BP reactivity to stress and found favorable results with attenuated hypertensive peaks. However, in addition to new studies being produced since then, responses to non-aerobic exercise are still unclear. Thus, the aim of the present systematic review with meta-analysis is to verify the acute effects of physical exercise on stress related BP reactivity in adults. The hypothesis is that the exercise will be able to mitigate these responses. 


\section{Methods}

This systematic review with meta-analysis followed PRISMA guidelines [13,14], had its protocol published (available from: dx.doi.org/10.17504/protocols.io.bhw3j7gn) [15] and was registered on "PROSPERO" (CRD42020194353).

\section{Eligibility criteria}

Studies with the following characteristics were eligible: 1) Population: human, both sexes, adults (i.e. $>18$ years), regardless of health or training status; 2) Intervention: a session of physical exercise; 3) Control: a session without exercise; 4) Outcome of interest: BP reactivity under stress (peak BP during a stress test or BP variation from basal levels); 5) Languages: English, Portuguese or Spanish; 6) Study designs: randomized clinical trials or crossovers; 7) Publication dates: no time limit; 8) Other characteristics: in studies with more than two intervention arms, only comparisons with the control group were considered, dividing the control sample proportionately to avoid sample duplication in the final analysis

\section{Search strategy}

The searches were performed on March 17th /2021, in digital databases (PUBMED, LILACS, EMBASE and Psyclnfo). Also, in the reference lists of the included studies, and through manual search in other websites ("https://core.ac.uk/" and "https://scholar.google.com/"). The search was organized into the following categories of terms: exercise intervention, BP and stressors. Parentheses and Boolean operators were used to organize the terms. All included terms are shown in the Supplementary table S1 and the flow diagram is shown in Fig. 1.

\section{Screening and data extraction process}

During the process of screening (title and abstract, and full-text stages), data extraction and risk of bias assessment, the studies were evaluated in duplicate by independent reviewers. After checking the responses, the reviewer's disagreements were resolved by consensus or by a third reviewer when necessary. The reviewer's agreement was estimated from Cohen's kappa in both full text screening $(\mathrm{K}=$ $0.631 ; p<0.001 ; 13$ desagreements were resolved by a third reviewer) and risk of bias assessment stages $(\kappa=0.877 ; p<0.001)$.

Before the data extraction phase, one of the reviewers standardized codes for all studies included in the following analyzes. Thus, each reviewer independently filled an electronic datasheet detailing the characteristics of the studies and the data was compared to assess agreement and identify errors. This datasheet included: identification code, author last name, publication year, language, study design, participants sexes and respective sample sizes, participants health and fitness status, age, hypertension status, other comorbidities, other relevant participants characteristics, exercise intensity, exercise volume (measured in minutes), exercise mode (aerobic, resistance, combined or yoga), stressor test, BP measure device/technique, and BP reactivity measures (sample sizes, mean and standard deviation. If other types of measures were reported, the mean and standard deviation were requested from the authors and in 
case of null or negative answers the results were transformed when possible). When there was not sufficient data for meta-analysis, the authors were contacted requesting these data. Studies in which the data are presented without numerical description, it was extracted through a web-based software (https://automeris.io/WebPlotDigitizer).

\section{Statistical analysis}

Pooled estimates were calculated using standardized mean differences (SMD) with confidence intervals $(95 \% \mathrm{Cl})$, using " $\mathrm{R}$ " programming language through the packages "meta" [16] and "metafor" [17]. In studies with multiple stressors, we used the mean and pooled dispersion between the stressors. The heterogeneity was measured by Kendall's tau and $\mathrm{I}^{2}$. Due to the different characteristics of interventions, population, and stress tests, we selected a random-effects approach using the Hunter Smith method to summarize the metanalytic results.

The sensitivity analysis was done through the search for outliers and influential points using externally standardized residuals, difference in fits, covariance ratio and Cook's distance methods. In addition, subgroup analyzes by type of stressor, the number of stressors, participants sexes, exercise mode, and studies design were made. The individual study assessment of the risk of bias was conducted through "Risk of Bias 2.0" [18] and its graphical visualization by the "R" package "robvis" [19]. Publication bias analyzes was carried out through Egger's regression and Beggs asymmetry tests, and trim and fill funnel plots.

\section{Results}

\section{Studies characteristics}

Studies included 425 women, 401 men, and 20 individuals in which sex was not disclosed. In addition, of the 28 studies, only $3(11 \%)$ included hypertensive patients, 21 (75\%) had a mean age of less than 30 years, 4 (15\%) were from 30 to 40 years old, and only 3 (11\%) were over 40 years old. As for stress tests, we have as the most frequent the Stroop color and word test (13 studies), followed by cold pressor and arithmetic test (9 studies each), public speaking (3 studies), handgrip ( 2 studies), and Trier Social Stress Test, anger- recall interview and study task (1 study each).

As for exercise characteristics, 2 studies included intervention with Yoga (7\%), 3 (11\%) with resistance exercises and only 1 (4\%) with combined exercises, all the others focused on aerobic exercises.

Furthermore, the exercise sessions lasted between 3 and 120 minutes (average of 30-60 minutes). As for intensity, 1 study used self-selection, 3 used high intensity and all others used low to moderate intensity (50-85\% of the individual maximum).

Regarding experimental designs, 6 (22\%) studies used a randomized clinical trial approach, and 22 (78\%) adopted a crossover design. As the main results, 11 (39\%) studies demonstrated improvements in SBP, 13 (46\%) in DBP, and 7 (out of $11 ; 64 \%$ ) in MBP. The others had null results since no study has shown 
harmful BP reactivity effects of exercise. Besides that, four studies did not present data dispersion measures to be included in the meta-analysis [20-23]. The general characteristics of all studies are shown in Table 1. 
Table 1

Studies characteristics.

\begin{tabular}{|c|c|c|c|c|}
\hline Study & Population & Stress test & Exercise & Reactivity results \\
\hline$[24]^{\star}$ & $\begin{array}{l}\text { NT, } 23 \text { women } \\
+17 \text { men, } 22 \\
\text { years, athletes }\end{array}$ & $\begin{array}{l}\text { Arithmetic + } \\
\text { Stroop color } \\
+ \text { Public } \\
\text { speech }\end{array}$ & $\begin{array}{l}\text { Aerobic (Maximum } \\
\text { incremental test) }\end{array}$ & $\downarrow M B P$ \\
\hline [25] & $\begin{array}{l}\text { NT, } 11 \text { women } \\
+13 \text { men, } 22 \\
\text { years }\end{array}$ & Arithmetic & Yoga (30min) & $\leftrightarrow \mathrm{SBP} \leftrightarrow \mathrm{DBP}$ \\
\hline [26] & $\begin{array}{l}\text { Borderline } \mathrm{HT} \text {, } \\
8 \text { participants, } \\
41 \text { years }\end{array}$ & Stroop color & $\begin{array}{l}\text { Aerobic (treadmill, 60min, } 60 \% \\
\left.\text { VO }_{2 \max }\right)\end{array}$ & $\downarrow S B P \downarrow D B P \downarrow M B P$ \\
\hline$[27]^{\star}$ & $\begin{array}{l}\text { NT, } 24 \text { men, } \\
22 \text { years }\end{array}$ & $\begin{array}{l}\text { Cold pressor } \\
+ \text { Stroop } \\
\text { color + Public } \\
\text { speech }\end{array}$ & $\begin{array}{l}\text { Aerobic }(60 \mathrm{~min} \text { or } 120 \mathrm{~min} \\
\left.55 \% \mathrm{VO}_{2 \mathrm{max}}\right)\end{array}$ & $\begin{array}{l}\text { Cold pressor: \SBP } \\
\text { \DBP Other tests: } \\
\leftrightarrow \mathrm{SBP} \leftrightarrow \mathrm{DBP}\end{array}$ \\
\hline [28] & $\begin{array}{l}\text { NT, } 30 \text { men, } \\
21 \text { years }\end{array}$ & Stroop color & $\begin{array}{l}\text { Aerobic (20min, } 75-85 \% \\
\text { HR reserve) }\end{array}$ & $\leftrightarrow \mathrm{SBP} \leftrightarrow \mathrm{DBP}$ \\
\hline [29] & $\begin{array}{l}\text { NT, } 9 \text { women, } \\
25 \text { years }\end{array}$ & Cold pressor & $\begin{array}{l}\text { Yoga or Aerobic (20min, auto } \\
\text { select intensity) }\end{array}$ & $\leftrightarrow \mathrm{SBP} \leftrightarrow \mathrm{DBP}$ \\
\hline [30] & $\begin{array}{l}\text { NT, } 10 \text { women } \\
+10 \text { men, } 33 \\
\text { years }\end{array}$ & Cold pressor & $\begin{array}{l}\text { Combined (30min, } 75-85 \% \\
\left.\mathrm{HR}_{\max } \text { and } 50 \% 1 \mathrm{RM}\right)\end{array}$ & $\downarrow S B P \downarrow D B P$ \\
\hline [31] & $\begin{array}{l}\text { NT, } 7 \text { men, } 23 \\
\text { years }\end{array}$ & $\begin{array}{l}\text { Hand grip }+ \\
\text { Stroop color }\end{array}$ & Aerobic $\left(120 \mathrm{~min}, 50 \% \mathrm{VO}_{2 \max }\right)$ & $\leftrightarrow \mathrm{SBP} \leftrightarrow \mathrm{DBP}$ \\
\hline [32] & $\begin{array}{l}\text { NT, } 12 \text { men, } \\
23 \text { years }\end{array}$ & $\begin{array}{l}\text { Cold pressor } \\
+ \text { Stroop } \\
\text { color }\end{array}$ & $\begin{array}{l}\text { Aerobic (treadmill, 30min, 60\% } \\
\text { VO }_{2 \max } \text { ) }\end{array}$ & $\begin{array}{l}\text { Stroop Color: \SBP } \\
\text { \DBP } \downarrow \text { MBP Cold } \\
\text { pressor: } \leftrightarrow \text { SBP } \leftrightarrow \text { DBP } \\
\leftrightarrow \text { MBP }\end{array}$ \\
\hline [33] & $\begin{array}{l}\text { NT, } 48 \text { women, } \\
25-40 \text { years }\end{array}$ & $\begin{array}{l}\text { Stroop color } \\
\text { + Public } \\
\text { speech }\end{array}$ & Aerobic (40min, $70 \% \mathrm{HR}_{\text {reserve }}$ ) & $\downarrow S B P \downarrow D B P \downarrow M B P$ \\
\hline [34] & $\begin{array}{l}\mathrm{NT}+\mathrm{HT}, 18 \\
\text { women }+14 \\
\text { men, 47-51 } \\
\text { years }\end{array}$ & $\begin{array}{l}\text { Arithmetic }+ \\
\text { Cold pressor }\end{array}$ & $\begin{array}{l}\text { Aerobic (20min, } 60-70 \% \\
\left.\mathrm{HR}_{\max }\right)\end{array}$ & $\leftrightarrow \mathrm{SBP} \downarrow \mathrm{DBP} \leftrightarrow \mathrm{MBP}$ \\
\hline [35] & $\begin{array}{l}\text { NT, } 42 \text { women } \\
+48 \text { men, } 23 \\
\text { years }\end{array}$ & Arithmetic & $\begin{array}{l}\text { Aerobic (30min, } 50-55 \% \\
\left.\text { VO }_{2 \max } \text { or } 75-80 \% \mathrm{VO}_{2 \max }\right)\end{array}$ & $\begin{array}{l}\text { Both intensities: } \downarrow \text { SBP } \\
\text { } D \text { DBP }\end{array}$ \\
\hline
\end{tabular}

The age refers to the average. SBP: systolic blood pressure; DBP: diastolic blood pressure; MBP: mean blood pressure; HR: heart rate; HT: hypertensives; NT: normotensives; *: randomized clinical trials, the other studies are cross over designs. 


\begin{tabular}{|c|c|c|c|c|}
\hline Study & Population & Stress test & Exercise & Reactivity results \\
\hline [36] & $\begin{array}{l}\text { NT, } 6 \text { women } \\
+9 \text { men, } 26 \\
\text { years }\end{array}$ & Cold pressor & $\begin{array}{l}\text { Resistance (30min, } 40-60 \% \\
1 \mathrm{RM})\end{array}$ & $\leftrightarrow \mathrm{SBP} \downarrow \mathrm{DBP}$ \\
\hline [37] & $\begin{array}{l}\text { NT, } 18 \text { men, } \\
20 \text { years }\end{array}$ & Arithmetic & $\begin{array}{l}\text { Resistance (Eccentric } \\
\text { movement, 45min, 120\% 1RM) }\end{array}$ & $\leftrightarrow \mathrm{SBP} \leftrightarrow \mathrm{DBP}$ \\
\hline [38] & $\begin{array}{l}\text { NT, } 24 \text { women } \\
\text { (11 smokers), } \\
21 \text { years }\end{array}$ & $\begin{array}{l}\text { Cold pressor } \\
+ \text { Stroop } \\
\text { color }\end{array}$ & Aerobic (30min, $\left.50 \% \mathrm{VO}_{2 \text { peak }}\right)$ & $\leftrightarrow \mathrm{SBP} \leftrightarrow \mathrm{DBP} \leftrightarrow \mathrm{MBP}$ \\
\hline [39] & $\begin{array}{l}\mathrm{NT}+\mathrm{HT}, 12 \\
\text { women }+18 \\
\text { men, } 41 \text { years }\end{array}$ & Stroop color & Aerobic (53min, $\left.50 \% \mathrm{VO}_{2 \text { peak }}\right)$ & $\downarrow S B P \downarrow D B P$ \\
\hline [40] & $\begin{array}{l}\text { NT, } 11 \text { men, } \\
25 \text { years }\end{array}$ & Arithmetic & Aerobic (30min, $\left.70 \% \mathrm{HR}_{\max }\right)$ & $\leftrightarrow \mathrm{SBP} \leftrightarrow \mathrm{DBP} \leftrightarrow \mathrm{MBP}$ \\
\hline$[41]^{\star}$ & $\begin{array}{l}\text { NT, } 80 \text { women, } \\
18 \text { years }\end{array}$ & Stroop color & $\begin{array}{l}\text { Aerobic }(10 \mathrm{~min} \text { or } 25 \mathrm{~min} \text { or } \\
\left.40 \mathrm{~min}, 70 \% \mathrm{HR}_{\text {reserve }}\right)\end{array}$ & $\leftrightarrow \mathrm{SBP} \downarrow \mathrm{DBP} \downarrow \mathrm{MBP}$ \\
\hline [42] & $\begin{array}{l}\text { NT, } 12 \\
\text { participants, } \\
31 \text { years }\end{array}$ & Stroop color & $\begin{array}{l}\text { Aerobic }\left(30 \mathrm{~min} \text { at } 50 \% \mathrm{VO}_{2 \max }\right. \\
\left.\text { or } 60 \mathrm{~min} \text { at } 80 \% \mathrm{VO}_{2 \max }\right)\end{array}$ & 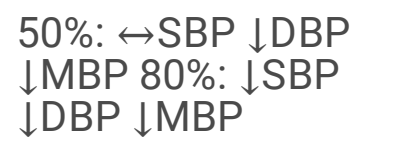 \\
\hline [43] & $\begin{array}{l}\text { NT, } 9 \text { men, } 32 \\
\text { years }\end{array}$ & $\begin{array}{l}\text { Hand grip }+ \\
\text { Stroop color } \\
+ \text { Arithmetic }\end{array}$ & Aerobic $\left(30 \mathrm{~min}, 60 \% \mathrm{VO}_{2 \max }\right)$ & $\leftrightarrow \mathrm{SBP} \leftrightarrow \mathrm{DBP}$ \\
\hline [44] & $\begin{array}{l}\text { NT, } 22 \text { women } \\
+4 \text { men, } 29 \\
\text { years }\end{array}$ & Stroop color & $\begin{array}{l}\text { Aerobic (Maximum } \\
\text { incremental test) }\end{array}$ & $\downarrow \mathrm{SBP} \leftrightarrow \mathrm{DBP} \leftrightarrow \mathrm{MBP}$ \\
\hline [45] & $\begin{array}{l}\text { NT, } 22 \text { men, } \\
23 \text { years }\end{array}$ & Cold pressor & $\begin{array}{l}\text { Aerobic ( } 30 \mathrm{~min} \text { at } 50-60 \\
\mathrm{HR}_{\text {reserve }} \text { or } 20 \mathrm{~min} \text { interval } \\
(4 \times 3 \mathrm{~min} / 2 \mathrm{~min}) \text { at } 80-90 \% \\
\left.\mathrm{HR}_{\text {reserve }}\right)\end{array}$ & $\leftrightarrow \mathrm{SBP} \leftrightarrow \mathrm{DBP}$ \\
\hline$[46]^{\star}$ & $\begin{array}{l}\text { NT, } 52 \text { women } \\
+27 \text { men, } 22 \\
\text { years }\end{array}$ & $\begin{array}{l}\text { Anger-recall } \\
\text { interview }\end{array}$ & Aerobic (3min, walking) & $\leftrightarrow \mathrm{SBP} \leftrightarrow \mathrm{DBP}$ \\
\hline \multirow[t]{2}{*}{ [47] } & $\begin{array}{l}\text { NT, } 40 \text { men, } \\
26 \text { years }\end{array}$ & Cold pressor & $\begin{array}{l}\text { Resistance ( } 30 \mathrm{~min} \text { or } 50 \mathrm{~min} \text { at } \\
70 \% 1 \mathrm{RM})\end{array}$ & $\underset{\leftrightarrow \mathrm{MBP}}{30 \mathrm{~min}:} \leftrightarrow \mathrm{SBP} \leftrightarrow \mathrm{DBP}$ \\
\hline & & & & $\begin{array}{l}\text { 50min: } \downarrow \mathrm{SBP} \downarrow \mathrm{DBP} \\
\downarrow \mathrm{MBP}\end{array}$ \\
\hline
\end{tabular}

The age refers to the average. SBP: systolic blood pressure; DBP: diastolic blood pressure; MBP: mean blood pressure; HR: heart rate; HT: hypertensives; NT: normotensives; *: randomized clinical trials, the other studies are cross over designs. 


\begin{tabular}{|c|c|c|c|c|}
\hline Study & Population & Stress test & Exercise & Reactivity results \\
\hline$[20]^{*}$ & $\begin{array}{l}\text { NT, } 15 \text { men, } \\
21 \text { years }\end{array}$ & Arithmetic & $\begin{array}{l}\text { Aerobic (Cycle, } 20 \mathrm{~min} \text { at } 25 \text { or } \\
100 \text { watts) }\end{array}$ & $\begin{array}{l}25 \text { watts: } \leftrightarrow \text { SBP } \\
\leftrightarrow \text { DBP } 100 \text { watts: } \\
\text { \SBP } \downarrow D B P\end{array}$ \\
\hline [21] & $\begin{array}{l}\text { NT, } 18 \text { women, } \\
\text { undergraduate }\end{array}$ & $\begin{array}{l}40 \text { minutes } \\
\text { of study }\end{array}$ & $\begin{array}{l}\text { Aerobic ( } 40 \mathrm{~min} \text { at } 60-80 \% \\
\left.\mathrm{HR}_{\max }\right)\end{array}$ & $\leftrightarrow \mathrm{SBP} \leftrightarrow \mathrm{DBP}$ \\
\hline$[22]^{*}$ & $\begin{array}{l}\text { NT, } 40 \text { women } \\
+40 \text { men, } 21 \\
\text { years }\end{array}$ & Arithmetic & $\begin{array}{l}\text { Aerobic (20min at moderate } \\
\text { intensity) }\end{array}$ & $\leftrightarrow \mathrm{SBP} \leftrightarrow \mathrm{DBP}$ \\
\hline [23] & $\begin{array}{l}\text { NT, } 10 \text { women } \\
+13 \text { men, } 24 \\
\text { years }\end{array}$ & $\begin{array}{l}\text { Trier Social } \\
\text { Stress Test }\end{array}$ & Aerobic $\left(30 \mathrm{~min}, 70 \% \mathrm{VO}_{2 \text { peak }}\right)$ & $\leftrightarrow \mathrm{SBP}$ \\
\hline \multicolumn{5}{|c|}{$\begin{array}{l}\text { The age refers to the average. SBP: systolic blood pressure; DBP: diastolic blood pressure; MBP: mear } \\
\text { blood pressure; HR: heart rate; HT: hypertensives; NT: normotensives; *: randomized clinical trials, the } \\
\text { other studies are cross over designs. }\end{array}$} \\
\hline
\end{tabular}

\section{Meta-analysis results}

Among 24 studies included in meta-analysis, 8 presented multiple possible comparisons according to the exercise mode [29], exercise volume [27, 41, 47], exercise intensity [42, 45], parents smoking habit [28], or participants smoking habit [38]. Besides that, 22 studies demonstrate results for SBP (32 comparisons), 23 for DBP (33 comparisons) and 11 for MBP (16 comparisons). The forest plots of SBP, DBP and MBP reactivity are present in Figs. 2,3 and 4, respectively. We found small but favorable results to exercise in both SBP (SMD = -0.35 [-0.46; -0.23$]$, representing average reductions of $3.8 \pm 3.5 \mathrm{mmHg})$, DBP (SMD = $-0.49[-0.68 ;-0.30]$, representing average reductions of $3.1 \pm 3.6 \mathrm{mmHg})$ and $\mathrm{MBP}$ reactivity $(\mathrm{SMD}=-0.48$ $[-0.70 ;-0.26]$, representing average reductions of $4.1 \pm 3.0 \mathrm{mmHg}$ ). We also highlight that 20 of the studies were carried out in healthy non-athlete individuals aged up to 40 years. Thus, by isolating the analyzes for this population, we maintain the results like the above for SBP (SMD $=-0.30[-0.43 ;-0.18])$, DBP $(S M D=$ $-0.43[-0.61 ;-0.25])$, and MBP (SMD $=-0.37[-0.54 ;-0.20])$.

Sensitivity analyzes showed that 4 studies $[26,27,34,44]$ can be outliers and/or influential points in DBP and 1 study [26] in SBP and MBP reactivity. New analysis disregarding these studies showed a DBP effect size of -0.30 [-0.43; -0.17$]$, a SBP effect size of $-0.34[-0.45 ;-0.123]$ and a MBP effect size of -0.44 $[-0.62 ;-0.27]$. Subgroup sensitivity analyzes were performed in SBP and DBP, but none of these analyses reported significant differences between subgroups, either comparing: study design, participants sex, exercise mode, stress type or number of stressors. The summary of these analyzes can be seen in Table 2. 
Table 2

Summary of sensibility analysis for blood pressure responsiveness.

\begin{tabular}{llllll}
$\begin{array}{llll}\text { Subgroup } \\
\text { variables }\end{array}$ & \multicolumn{2}{l}{ Effect size } & & & \\
\cline { 2 - 5 } & SMD & $95 \% \mathrm{Cl}$ & $\begin{array}{l}\text { Weight } \\
(\%)\end{array}$ & $k$ & $\mathrm{~m}$
\end{tabular}

\section{Subgroup differences $p$}

Heterogeneity

$\dot{i}^{2} \quad \tau^{2} \quad Q$

(\%)

\section{SBP}

\section{Sex}

\begin{tabular}{|c|c|c|c|c|c|c|c|c|}
\hline Men & -0.35 & $\begin{array}{l}{[-0.52} \\
-0.17]\end{array}$ & 36.7 & 9 & $13 \quad 0.25$ & 0 & 0.0000 & 11.39 \\
\hline Women & -0.36 & $\begin{array}{l}{[-0.59} \\
-0.13]\end{array}$ & 21.2 & 4 & 8 & 0 & 0.0000 & 6.73 \\
\hline Both & -0.28 & $\begin{array}{l}{[-0.49 ;} \\
-0.07]\end{array}$ & 37.5 & 7 & 8 & 44 & 0.0289 & 12.42 \\
\hline Undefined & -0.85 & $\begin{array}{l}{[-1.37 ;} \\
-0.34]\end{array}$ & 4.6 & 2 & 3 & 32 & 0.0000 & 2.95 \\
\hline Overall & -0.35 & $\begin{array}{l}{[-0.46} \\
-0.23]\end{array}$ & 100 & 22 & 32 & 17 & 0.0145 & 37.33 \\
\hline
\end{tabular}

\section{Exercise mode}

Yoga

0.15

$[-0.33 ;$
$0.64]$

4.9

22

0.22

$0 \quad 0.0000$

0.52

Aerobic

$\left.\begin{array}{ll}-0.37 \quad[-0.50 ; \\ -0.24\end{array}\right]$

76.9

$17 \quad 25$

15

0.0118

28.37

Combined

$-0.30$

$[-0.92$;

3.0

11

0.33 ]

Resistance

$-0.40$

$[-0.66$;

$-0.13]$

15.3

34

$\begin{array}{lll}24 & 0.000 \quad 3.92\end{array}$

Overall

$\left.\begin{array}{ll}-0.35 & {[-0.46 ;} \\ -0.23\end{array}\right]$

100

$23 \quad 32$

$\begin{array}{lll}17 & 0.0145 & 37.33\end{array}$

\section{Study design}

\begin{tabular}{|c|c|c|c|c|c|c|c|c|c|}
\hline RCT & -0.39 & $\begin{array}{l}{[-0.67} \\
-0.11]\end{array}$ & 19.2 & 3 & 6 & 0.73 & 37 & 0.0291 & 7.9 \\
\hline Cross over & -0.34 & $\begin{array}{l}{[-0.46 ;} \\
-0.21]\end{array}$ & 80.8 & 19 & 26 & & 15 & 0.0111 & 29.34 \\
\hline
\end{tabular}

Include physical stressor: studies that used only physical stressors or in conjunction with mental stressors; SBP: systolic blood pressure; DBP: diastolic blood pressure; SMD: effect size by standardized mean differences; $\mathrm{Cl}$ : credible interval; $\mathrm{k}$ : number of studies; m: number of comparisons; *: $p<0.05$. 


\begin{tabular}{|c|c|c|c|c|c|c|c|c|c|}
\hline \multirow{2}{*}{$\begin{array}{l}\text { Subgroup } \\
\text { variables }\end{array}$} & \multicolumn{5}{|c|}{ Effect size } & \multirow{2}{*}{$\begin{array}{l}\text { Subgroup } \\
\text { differences p }\end{array}$} & \multicolumn{3}{|c|}{ Heterogeneity } \\
\hline & SMD & $95 \% \mathrm{Cl}$ & $\begin{array}{l}\text { Weight } \\
\text { (\%) }\end{array}$ & $\mathbf{k}$ & m & & $\begin{array}{l}\mathbf{i}^{2} \\
(\%)\end{array}$ & $\mathrm{T}^{2}$ & $\mathbf{Q}$ \\
\hline Overall & -0.35 & $\begin{array}{l}{[-0.46} \\
-0.23]\end{array}$ & 100 & 22 & 32 & & 17 & 0.0145 & 37.33 \\
\hline \multicolumn{10}{|c|}{ Stressor type } \\
\hline Mental & -0.31 & $\begin{array}{l}{[-0.47} \\
-0.14]\end{array}$ & 56.3 & 11 & 16 & 0.74 & 31 & 0.0262 & 21.68 \\
\hline Physical & -0.37 & $\begin{array}{l}{[-0.58} \\
-0.15]\end{array}$ & 24.7 & 5 & 8 & & 0 & 0.0000 & 6.82 \\
\hline Both & -0.42 & $\begin{array}{l}{[-0.68} \\
-0.17]\end{array}$ & 19.0 & 6 & 8 & & 16 & 0.0053 & 8.34 \\
\hline Overall & -0.35 & $\begin{array}{l}{[-0.46} \\
-0.23]\end{array}$ & 100 & 22 & 32 & & 17 & 0.0145 & 37.33 \\
\hline \multicolumn{10}{|c|}{ Number of stressors } \\
\hline Multiple & -0.35 & $\begin{array}{l}{[-0.60} \\
-0.11]\end{array}$ & 28.4 & 8 & 10 & 0.94 & 38 & 0.0452 & 14.45 \\
\hline Unique & -0.34 & $\begin{array}{l}{[-0.47} \\
-0.22]\end{array}$ & 71.6 & 14 & 22 & & 8 & 0.0029 & 22.77 \\
\hline Overall & -0.35 & $\begin{array}{l}{[-0.46} \\
-0.23]\end{array}$ & 100 & 22 & 32 & & 17 & 0.0145 & 37.33 \\
\hline \multicolumn{10}{|l|}{ DBP } \\
\hline \multicolumn{10}{|l|}{ Sex } \\
\hline Men & -0.49 & $\begin{array}{l}{[-0.85} \\
-0.12]\end{array}$ & 38.4 & 9 & 13 & 0.09 & 77 & 0.3221 & $51.37 *$ \\
\hline Women & -0.35 & $\begin{array}{l}{[-0.65} \\
-0.06]\end{array}$ & 23.1 & 4 & 8 & & 42 & 0.0551 & 11.99 \\
\hline Both & -0.42 & $\begin{array}{l}{[-0.72} \\
-0.12]\end{array}$ & 31.8 & 8 & 9 & & 74 & 0.1360 & $31.35^{\star}$ \\
\hline Undefined & -1.16 & $\begin{array}{l}{[-1.72} \\
-0.59]\end{array}$ & 6.7 & 2 & 3 & & 39 & 0.0217 & 3.29 \\
\hline Overall & -0.49 & $\begin{array}{l}{[-0.68} \\
-0.30]\end{array}$ & 100 & 23 & 33 & & 70 & 0.1979 & 106.09* \\
\hline \multicolumn{10}{|c|}{ Exercise mode } \\
\hline $\begin{array}{l}\text { Include phy } \\
\text { stressors; S } \\
\text { standardize } \\
\star:: p<0.05 \text {. }\end{array}$ & $\begin{array}{l}\text { stress } \\
\text { ystoli } \\
\text { an dif }\end{array}$ & $\begin{array}{l}\text { studie } \\
\text { lood pr } \\
\text { ences; }\end{array}$ & $\begin{array}{l}\text { t used } \\
\text { re; DBF } \\
\text { redible }\end{array}$ & y ph & & $\begin{array}{l}\text { tressors or in } \\
\text { d pressure; } \\
\text { ber of studies }\end{array}$ & junc & $\begin{array}{l}n \text { with m } \\
\text { size by } \\
\text { er of con }\end{array}$ & atal \\
\hline
\end{tabular}




\begin{tabular}{|c|c|c|c|c|c|c|c|c|c|}
\hline \multirow{2}{*}{$\begin{array}{l}\text { Subgroup } \\
\text { variables }\end{array}$} & \multicolumn{5}{|c|}{ Effect size } & \multirow{2}{*}{$\begin{array}{l}\text { Subgroup } \\
\text { differences p }\end{array}$} & \multicolumn{3}{|c|}{ Heterogeneity } \\
\hline & SMD & $95 \% \mathrm{Cl}$ & $\begin{array}{l}\text { Weight } \\
(\%)\end{array}$ & k & $\mathrm{m}$ & & 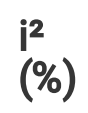 & $\tau^{2}$ & $\mathbf{Q}$ \\
\hline Yoga & -0.77 & $\begin{array}{l}{[-1.27 ;} \\
-0.27]\end{array}$ & 5.5 & 2 & 2 & \multirow[t]{5}{*}{0.67} & 0 & 0.0000 & 0.2 \\
\hline Aerobic & -0.48 & $\begin{array}{l}{[-0.71 ;} \\
-0.24]\end{array}$ & 77.6 & 18 & 26 & & 74 & 0.2440 & $96.83^{\star}$ \\
\hline Combined & -0.30 & $\begin{array}{l}{[-0.93} \\
0.32]\end{array}$ & 3.2 & 1 & 1 & & - & - & - \\
\hline Resistance & -0.50 & $\begin{array}{l}{[-0.86} \\
-0.14]\end{array}$ & 13.6 & 3 & 4 & & 57 & 0.0558 & 7.01 \\
\hline Overall & -0.49 & $\begin{array}{l}{[-0.68} \\
-0.30]\end{array}$ & 100 & 24 & 33 & & 70 & 0.1979 & 106.09* \\
\hline \multicolumn{10}{|c|}{ Study design } \\
\hline RCT & -0.79 & $\begin{array}{l}{[-1.45} \\
-0.12]\end{array}$ & 19.2 & 3 & 6 & \multirow[t]{3}{*}{0.30} & 88 & 0.5855 & $40.55^{\star}$ \\
\hline Cross over & -0.42 & $\begin{array}{l}{[-0.60} \\
-0.24]\end{array}$ & 80.8 & 20 & 27 & & 58 & 0.1130 & $62.08^{\star}$ \\
\hline Overall & -0.49 & $\begin{array}{l}{[-0.68} \\
-0.30]\end{array}$ & 100 & 23 & 33 & & 70 & 0.1979 & 106.09* \\
\hline \multicolumn{10}{|c|}{ Stressor type } \\
\hline Mental only & -0.30 & $\begin{array}{l}{[-0.48} \\
-0.12]\end{array}$ & 53.8 & 12 & 17 & \multirow[t]{4}{*}{0.19} & 44 & 0.0495 & $28.44^{\star}$ \\
\hline $\begin{array}{l}\text { Physical } \\
\text { only }\end{array}$ & -0.56 & $\begin{array}{l}{[-0.82} \\
-0.29]\end{array}$ & 24.5 & 5 & 8 & & 39 & 0.0413 & 11.47 \\
\hline Both & -0.72 & $\begin{array}{l}{[-1.38} \\
-0.05]\end{array}$ & 21.6 & 6 & 8 & & 86 & 0.7464 & $48.32^{\star}$ \\
\hline Overall & -0.49 & $\begin{array}{l}{[-0.68} \\
-0.30]\end{array}$ & 100 & 23 & 33 & & 70 & 0.1979 & 106.09* \\
\hline \multicolumn{10}{|c|}{ Number of stressors } \\
\hline Multiple & -0.69 & $\begin{array}{l}{[-1.18} \\
-0.20]\end{array}$ & 29.0 & 8 & 10 & \multirow[t]{2}{*}{0.23} & 83 & 0.4898 & $53.54^{\star}$ \\
\hline Unique & -0.37 & $\begin{array}{l}{[-0.54 ;} \\
-0.20]\end{array}$ & 71.0 & 15 & 23 & & 47 & 0.0672 & $41.84^{\star}$ \\
\hline
\end{tabular}

Include physical stressor: studies that used only physical stressors or in conjunction with mental stressors; SBP: systolic blood pressure; DBP: diastolic blood pressure; SMD: effect size by standardized mean differences; Cl: credible interval; k: number of studies; m: number of comparisons; *: $p<0.05$. 


\begin{tabular}{|c|c|c|c|c|c|c|c|c|c|}
\hline \multirow{2}{*}{$\begin{array}{l}\text { Subgroup } \\
\text { variables }\end{array}$} & \multicolumn{5}{|c|}{ Effect size } & \multirow{2}{*}{$\begin{array}{l}\text { Subgroup } \\
\text { differences p }\end{array}$} & \multicolumn{3}{|c|}{ Heterogeneity } \\
\hline & SMD & $95 \% \mathrm{Cl}$ & $\begin{array}{l}\text { Weight } \\
\text { (\%) }\end{array}$ & k & m & & $\begin{array}{l}\mathrm{i}^{2} \\
(\%)\end{array}$ & $\tau^{2}$ & $\mathbf{Q}$ \\
\hline Overall & -0.49 & $\begin{array}{l}{[-0.68 ;} \\
-0.30]\end{array}$ & 100 & 23 & 33 & & 70 & 0.1979 & 106.09* \\
\hline \multicolumn{10}{|c|}{$\begin{array}{l}\text { Include physical stressor: studies that used only physical stressors or in conjunction with mental } \\
\text { stressors; SBP: systolic blood pressure; DBP: diastolic blood pressure; SMD: effect size by } \\
\text { standardized mean differences; Cl: credible interval; } k \text { : number of studies; } m \text { : number of comparisons; } \\
\star:: p<0.05 \text {. }\end{array}$} \\
\hline
\end{tabular}

\section{Bias assessment}

In general, studies present a low to moderate risk of bias in all domains (Fig. 5). Just one study mentions the previous existence of protocols or clinical study records, making it difficult to analyze bias related to the selection of reported results. None of the studies reported intention-to-treat analysis, conflicts of interest or participants were blinded to interventions, what is expected in physical exercise interventions and does not seem to be a major problem in this type of intervention [48]. The publication bias tests showed no asymmetries in the funnel plot for SBP (Egger regression $p=0.575$; Begg's test $p=0.697$ ), DBP (Egger regression $p=0.450$; Begg's test $p=0.321$ ) or MBP reactivity (Egger regression $p=0.733$; Begg's test $p=0.528$ ). However, six omitted results are expected by trim and fill funnel plots only in SBP (Fig. 6).

\section{Discussion}

Our main results were that $61 \%$ (17 out of 28 ) of the included studies reported attenuated BP peaks (either in SBP, DPB and/or MBP) after acute exercise and none showed deleterious results from the exercise. The metanalytic results suggest that acute exercise attenuates $\mathrm{BP}$ reactivity to stress. This effect occurred mutually in SBP (SMD $=-0.35[-0.46 ;-0.23])$, DBP (SMD $=-0.49[-0.68 ;-0.30])$ and MBP $(S M D=-0.48[-0.70 ;-0.26])$ in magnitudes similar to previous meta-analyze about the effects of acute aerobic exercise (SBP Effect size $=0.38$; DBP Effect size $=0.40$ ) [12]. Besides that, only $22 \%$ of the studies included non-aerobic exercises which make the results for these exercise mode difficult to generalize. Lastly, there is a scarcity of studies with hypertensive individuals (11\%) and with a population over 40 years old $(11 \%)$.

In this sense, we reaffirm the need for studies with high cardiovascular risk patients, since these responses contribute to the construction of the clinical picture of these patients and may indicate an increase in left ventricular mass [9], augmented carotid atherosclerosis [49], increased risk of cardiovascular mortality [50], development of hypertension [11], and an increased risk of developing several cardiovascular diseases [2,4]. We also extend this need for studies with the elderly, who, in addition to having the aforementioned advantages for having a high incidence of cardiovascular diseases [51], seem to have very promising responses when compared to younger people [52], so studies 
exploring specific age stratus are needed. We also emphasize that, in addition to expanding and confirming favorable responses to aerobic exercise [12], the present study is, as far as we know, the first to demonstrate positive meta-analytic effects of resistance exercise in BP reactivity. It is worth mentioning that these results are anchored in a smaller volume of evidence, and should be interpreted with caution, but it provides an optimistic direction for future studies with this exercise mode.

Regarding intervention characteristics, studies that compare different exercise loads showed mixed results. As an example, three studies evaluated different exercise intensities and one was favorable to higher intensities [20], another obtained a very discreet advantage at greater intensities [42], and the latter found no differences between groups [35]. Concerning exercise session duration, a study shows favorable effects of longer sessions [47], and the others found no differences [27, 41]. Finally, a study compared continuous aerobic exercise of moderate intensity with high intensity interval exercise and also found no significant differences [45]. Thus, evidence on differences arising from the characteristics of exercise load control is still scarce, therefore a meta-analysis clustering intensity groups was not possible. However, the evidence is greater in moderate exercises for 30 to 60 minutes.

Overall, when exploring studies heterogeneity, we found that reductions in peak DBP appear to be more heterogeneous than those in SBP. In addition, the greatest effects found are usually in subgroups with fewer studies, and most of the heterogeneity seems to be driven by studies published before the year 2000. So, regarding the effect on DBP response, several results must be highlighted. The first is that, in sex comparisons, the high heterogeneity in men $\left(\mathrm{i}^{2}=77\right)$ draws attention and seems to be explained by Ebbesen et. al study [27]. This study has a very favorable effect on exercise and is not overlapping with other studies, and with its omission we have important reductions in heterogeneity and effect size $\left(\mathrm{i}^{2}=0\right.$; $\mathrm{SMD}=-0.28[-0.47 ;-0.09]$ ). The large volume of exercise in this study (from 60 to $120 \mathrm{~min}$ ) may also explain this difference. Also, there is an important heterogeneity related to studies that include both sexes $\left(\mathrm{i}^{2}=74 \%\right)$. A point that still draws a lot of attention in comparisons by sex, is the large effect size related to studies without a defined sex $(-1.16[-1.72 ;-0.59])$. However, this subgroup has only 2 studies, and one of them [26] has an exceptionally large effect $(-2.06[-3.28 ;-0.85])$.

Besides that, there is high heterogeneity in studies with aerobic exercise $\left(i^{2}=74 \%\right)$. The main characteristics of these studies that may explain their differences to the others in the subgroup are the inclusion of hypertensive patients [26, 34], the high volume of exercise (from 60 to $120 \mathrm{~min}$ ) [27] and the self-selected exercise intensity strategy [29]. Regarding the studies with resistance exercises, the heterogeneity is significantly reduced (from $\mathrm{i}^{2}=54 \%$, to $\mathrm{i}^{2}=0 \%$, with SMD $=-0.65[-0.95 ;-0.36]$ ) with the omission of one study [37]. This heterogeneity might be explained by the alternative training with an intensity much higher than that of other studies (eccentric phase training at $120 \%$ of 1 repetition maximum test). Furthermore, the high heterogeneity $\left(i^{2}=88 \%\right)$ and the moderate effect size $(-0.79[-1.45$; -0.12]) in studies with RCT design are also noteworthy. In this regard, when we remove the study from Ebbesen et. al [27], drastically reduces the heterogeneity and effect size of the subgroup $\left(\mathrm{i}^{2}=0 \%\right.$; $\mathrm{SMD}=$ $-0.19[-0.49 ; 0.11]$ ). This might be explained by the large volume of exercise in this study (from 60 to 120 $\mathrm{min}$ ) compared to the others in the subgroup (3 to 45 minutes). 
Considering types of stressors, there are moderate effects in studies that present physical tests (isolated or both), but mental tests alone have small effects. This may indicate greater effects of exercise in situations of physical stress than in situations of mental stress. Furthermore, the high heterogeneity of the group with associated physical and mental stressors $\left(i^{2}=86 \%\right)$ draws attention, but it was expected due to the heterogeneity of the stress tests. However, 2 studies stand out in this subgroup for not having results that overlap with the others [27,34]. The main characteristics of these studies that can explain their differences in relation to the others in the subgroup are the large volume of training (from 60 to 120 $\mathrm{min}$ ) in one study [27] and the inclusion of hypertensive patients in the other [34]. The omission of these studies reduces the heterogeneity $\left(\mathrm{i}^{2}=0 \%\right)$ and the effect size $(0.00[-0.35 ; 0.35])$, generating a situation in which studies with isolated effects have effects weak to moderate while in tests with both associated have null effects. These studies also have no overlap with the others from the multiple stressors subgroup, and their omission generates a reduction in heterogeneity (from $\mathrm{i}^{2}=83 \%$, to $\mathrm{i}^{2}=0 \%$ ) and in effect size (from 0.69 [-1.18; -0.20$]$, to $-026[-0.50 ;-0.01]$ ).

Another source of heterogeneity could be the fact that several stress tests were used, from classically standardized and widely used protocols such as the Cold pressor test [53] to less restricted but with greater ecological validity as studying situations [21]. In this sense, we believe that a convergence of these characteristics is necessary, to combine sufficient standardization of methods with greater continuity with the stress experienced in daily life [5]. Thus, studies with multiple stressors such as the Trier Social Stress Test (that includes public speaking with simulated job interview and arithmetic task) and the Maastricht Acute Stress Test (that includes cold pressure stress, negative feedback and arithmetic task) seem to be good alternatives for future studies [5].

As the types of stressors, their mechanisms of action are also diverse. So, when a stressful situation is imposed, it generates a response that includes diverse mechanisms [1-3], such as: neural-network (specially salience, executive control, and default mode networks) [54, 55], autonomic system [56, 57], catecholamines [3,58], cortisol $[59,60]$, and opioids/ $\beta$ endorphin $[61,62]$. So, the isolated and interaction effects [63] of these mechanisms may explain the BP reactivity to stress [3,64]. Exercise, in turn, seems to mitigate stress reactivity by reducing vascular resistance [34], norepinephrine [65] and hypothalamic pituitary-adrenal axis responses [66], in addition to causing increased $\beta 2$-mediated vasodilation [65] and levels of endorphins [67]. Finally, there are also psychosocial effects of exercise such as improved selfefficacy and distraction from negative feelings [68].

It should be emphasized that the present review has some limitations, such as the multiplicity of stress tests and exercise prescrition, which makes difficult generalize the results. Besides that, these results are mostly in healthy and young populations and therefore cannot be easily generalized to populations with different health conditions. Thus, in future studies, we encourage the research of stressors similar to everyday life, involving different situations, sensations, emotions, and specially extended stressors like those found in sports, social fragility, and scholar/work environment. In this sense, we highlight a study [21], which despite achieving null results, has an interesting approach with great ecological validity (40 minutes studying with undergraduate students). Finally, we also encourage studies that allow a better 
understanding of exercise load control (e.g., intensity, volume), and in older populations with different morbidities, that can help to improve individual intervention strategies.

\section{Conclusion}

In summary, acute physical exercise lowers SBP, DBP and MBP reactivity to stressor tests. However, given the small magnitude of effects, the clinical relevance of this result must be interpreted with caution and be better explored. Further studies would help understand the effect of different exercise modalities to apply to different clinical profiles, helping exploring the clinical application of this screening tool.

\section{Declarations}

Author contributions statement: IMM had the idea for the article. IMM, ALA, PABR and GMP participated in the study planning and structuring. IMM and ALA performed the literature search and data analysis. PABR and GMP drafted and critically revised the work. IMM, ALA, PABR and GMP approved the final version of the manuscript.

Competing interests: The author(s) declare no competing interests.

Data availability: The datasets generated during and/or analysed during the current study are available from the corresponding author on reasonable request.

\section{References}

1. Rab, S. L. \& Admon, R. Parsing inter- and intra-individual variability in key nervous system mechanisms of stress responsivity and across functional domains. Neurosci. Biobehav. Rev. (2020). doi:10.1016/j.neubiorev.2020.09.007

2. Huang, C.-J., Webb, H. E., Zourdos, M. C. \& Acevedo, E. O. Cardiovascular reactivity, stress, and physical activity. Front. Physiol. 4, 314 (2013).

3. Chrousos, G. Stress and disorders of the stress system. Nat. Rev. Endocrinol. 5, 374-381 (2009).

4. Turner, A. I. et al. Psychological stress reactivity and future health and disease outcomes: A systematic review of prospective evidence. Psychoneuroendocrinology 114, 104599 (2020).

5. Bali, A. \& Jaggi, A. S. Clinical experimental stress studies: methods and assessment. Rev. Neurosci. 26, (2015).

6. Arima, H., Barzi, F. \& Chalmers, J. Mortality patterns in hypertension. J. Hypertens. 29, S3-S7 (2011).

7. Muntner, P. Response to Letter to editor "2017 ACC/AHA/AAPA/ABC/ACPM/AGS/APhA/ASH/ASPC/NMA/PCNA Guideline for the Prevention, Detection, Evaluation, and Management of High Blood Pressure in Adults". J. Am. Soc. Hypertens. 12, 239 (2018). 
8. Halliwill, J. R., Buck, T. M., Lacewell, A. N. \& Romero, S. A. Postexercise hypotension and sustained postexercise vasodilatation: What happens after we exercise? Exp. Physiol. 98, 7-18 (2013).

9. Georgiades, A., Lemne, C., de Faire, U., Lindvall, K. \& Fredrikson, M. Stress-Induced Laboratory Blood Pressure in Relation to Ambulatory Blood Pressure and Left Ventricular Mass Among Borderline Hypertensive and Normotensive Individuals. Hypertension 28, 641-646 (1996).

10. Wood, D. L., Sheps, S. G., Elveback, L. R. \& Schirger, A. Cold pressor test as a predictor of hypertension. Hypertension 6, 301-306 (1984).

11. Matthews, K. A., Woodall, K. L. \& Allen, M. T. Cardiovascular reactivity to stress predicts future blood pressure status. Hypertension 22, 479-485 (1993).

12. Hamer, M., Taylor, A. \& Steptoe, A. The effect of acute aerobic exercise on stress related blood pressure responses: A systematic review and meta-analysis. Biol. Psychol. 71, 183-190 (2006).

13. Moher, D., Liberati, A., Tetzlaff, J. \& Altman, D. G. Preferred Reporting Items for Systematic Reviews and Meta-Analyses: The PRISMA Statement. PLoS Med. 6, e1000097 (2009).

14. Page, M. et al. The PRISMA 2020 statement: an updated guideline for reporting systematic reviews. 1-36 (2020). doi:10.31222/osf.io/v7gm2

15. Mariano, I. M., Amaral, A. L. \& Puga, G. M. Protocol of a systematic review with meta-analysis: Acute effects of physical exercise on blood pressure responsiveness to non-cardiopulmonary stress tests. (2020). doi:10.17504/protocols.io.bhw3j7gn

16. Balduzzi, S., Rücker, G. \& Schwarzer, G. How to perform a meta-analysis with R: a practical tutorial. Evid. Based Ment. Heal. 22, 153-160 (2019).

17. Viechtbauer, W. Conducting meta-analyses in R with the metafor package. J. Stat. Softw. 36, 1-48 (2010).

18. Higgins, J. P., Savović, J., Page, M. J. \& Sterne, J. A. RoB 2: A revised Cochrane risk-of-bias tool for randomized trials. BMJ (in Press. 1-24 (2019). at <https://methods.cochrane.org/>

19. McGuinness, L. A. \& Higgins, J. P. T. Risk-of-bias VISualization (robvis): An R package and Shiny web app for visualizing risk-of-bias assessments. Res. Synth. Methods jrsm.1411 (2020).

doi:10.1002/jrsm.1411

20. Roy, M. \& Steptoe, A. The inhibition of cardiovascular responses to mental stress following aerobic exercise. Psychophysiology 28,689-700 (1991).

21. FLORY, J. D. \& HOLMES, D. S. EFFECTS OF AN ACUTE BOUT OF AEROBIC EXERCISE ON CARDIOVASCULAR AND SUBJECTIVE RESPONSES DURING SUBSEQUENT COGNITIVE WORK. J. PsychosomReasearricch 35, 225-230 (1991).

22. Roth, D. L. Acute emotional psychophysiological effects of aerobic exercise. Psychophysiology 26, (1989).

23. Leow, S. et al. The effect of antecedent exercise on the acute stress response and subsequent food consumption: a preliminary investigation. Physiol. Behav. 229, 113256 (2021). 
24. Bartholomew, J. B. Stress reactivity after maximal exercise: the effect of manipulated performance feedback in endurance athletes. J. Sports Sci. 18, 893-899 (2000).

25. Benvenutti, M. J. et al. A single session of hatha yoga improves stress reactivity and recovery after an acute psychological stress task-A counterbalanced, randomized-crossover trial in healthy individuals. Complement. Ther. Med. 35, 120-126 (2017).

26. Boone, J. B., Probst, M. M., Rogers, M. W. \& Berger, R. Postexercise hypotension reduces cardiovascular responses to stress. J. Hypertens. 11, 449-453 (1993).

27. Ebbesen, B. L., Prkachin, K. M., Mills, D. E. \& Green, H. J. Effects of acute exercise on cardiovascular reactivity. J. Behav. Med. 15, 489-507 (1992).

28. Hamer, M., Jones, J. \& Boutcher, S. H. Acute exercise reduces vascular reactivity to mental challenge in offspring of hypertensive families. J. Hypertens. 24, 315-320 (2006).

29. Monroe, D. C., Yin, J., McCully, K. K. \& Dishman, R. K. Yoga Aids Blood Pressure Recovery After Exposure of Forehead to Cold: A Pilot Study. Altern. Ther. Health Med. 24, 12-17 (2018).

30. Moreira, S. R., Lima, R. M., Silva, K. E. S. \& Simões, H. G. Combined exercise circuit session acutely attenuates stress-induced blood pressure reactivity in healthy adults. Brazilian J. Phys. Ther. 18, 3846 (2014).

31. Péronnet, F., Massicotte, D., Paquet, J. E., Brisson, G. \& de Champlain, J. Blood pressure and plasma catecholamine responses to various challenges during exercise-recovery in man. Eur. J. Appl. Physiol. Occup. Physiol. 58, 551-555 (1989).

32. Probst, M., Bulbulian, R. \& Knapp, C. Hemodynamic responses to the stroop and cold pressor tests after submaximal cycling exercise in normotensive males. Physiol. Behav. 62, 1283-1290 (1997).

33. Rejeski, W. J., Thompson, A., Brubaker, P. H. \& Miller, H. S. Acute exercise: buffering psychosocial stress responses in women. Health Psychol. 11, 355-362 (1992).

34. West, S. G. et al. Postexercise vasodilatation reduces diastolic blood pressure responses to stress. Ann. Behav. Med. 20, 77-83 (1998).

35. Alderman, B. L., Arent, S. M., Landers, D. M. \& Rogers, T. J. Aerobic exercise intensity and time of stressor administration influence cardiovascular responses to psychological stress.

Psychophysiology 44, 759-766 (2007).

36. Heffernan, K. S. et al. Carotid artery reactivity during sympathetic activation following acute resistance exercise. Clin. Auton. Res. Off. J. Clin. Auton. Res. Soc. 27, 417-421 (2017).

37. Paine, N. J. et al. Eccentric-exercise induced inflammation attenuates the vascular responses to mental stress. Brain. Behav. Immun. 30, 133-142 (2013).

38. Rooks, C. R., McCully, K. K. \& Dishman, R. K. Acute exercise improves endothelial function despite increasing vascular resistance during stress in smokers and nonsmokers. Psychophysiology 48, 1299-1308 (2011).

39. Santaella, D. F. et al. Aftereffects of exercise and relaxation on blood pressure. Clin. J. Sport Med. Off. J. Can. Acad. Sport Med. 16, 341-347 (2006). 
40. Someya, N., Ikemura, T. \& Hayashi, N. Effect of preceding exercise on cerebral and splanchnic vascular responses to mental task. J. Physiol. Anthropol. 31, 17 (2012).

41. Hobson, M. L. \& Rejeski, W. J. Does the Dose of Acute Exercise Mediate Psychophysiological Responses to Mental Stress? J. Sport Exerc. Psychol. 15, 77-87 (1993).

42. Rejeski, W. J., Gregg, E., Thompson, A. \& Berry, M. The Effects of Varying Doses of Acute Aerobic Exercise on Psychophysiological Stress Responses in Highly Trained Cyclists. J. Sport Exerc. Psychol. 13, 188-199 (1991).

43. Szabo, A. et al. Psychophysiological profiles in response to various challenges during recovery from acute aerobic exercise. Int. J. Psychophysiol. 14, 285-292 (1993).

44. Neves, F. J. et al. Hemodynamic mechanisms of the attenuated blood pressure response to mental stress after a single bout of maximal dynamic exercise in healthy subjects. Brazilian J. Med. Biol. Res. 45, 610-616 (2012).

45. Meireles, K. et al. Acute effects of moderate-intensity and high-intensity exercise on hemodynamic and autonomic reactivity to the cold pressor test in young adults with excess body weight. Blood Press. Monit. 82-88 (2020). doi:10.1097/MBP.0000000000000422

46. Routledge, F. S., McFetridge-Durdle, J. A., Macdonald, M., Breau, L. \& Campbell, T. The effect of exercise and distraction on blood pressure recovery following an anger-provoking stressor in normotensive young adults. J. Psychophysiol. 29, 45-54 (2015).

47. Lima da Silva, M. F. et al. The volume of resistance exercises influences blood pressure reactivity to stress. Rev. Bras. Med. do Esporte 21, 438-441 (2015).

48. Armijo-Olivo, S. et al. Blinding in Physical Therapy Trials and Its Association with Treatment Effects. Am. J. Phys. Med. Rehabil. 96, 34-44 (2017).

49. Kamarck, T. W. et al. Exaggerated Blood Pressure Responses During Mental Stress Are Associated With Enhanced Carotid Atherosclerosis in Middle-Aged Finnish Men. Circulation 96, 3842-3848 (1997).

50. Carroll, D. et al. Increased blood pressure reactions to acute mental stress are associated with 16year cardiovascular disease mortality. Psychophysiology 49, 1444-1448 (2012).

51. Yazdanyar, A. \& Newman, A. B. The Burden of Cardiovascular Disease in the Elderly: Morbidity, Mortality, and Costs. Clin. Geriatr. Med. 25, 563-577 (2009).

52. Uchino, B. N., Birmingham, W. \& Berg, C. A. Are older adults less or more physiologically reactive? A meta-analysis of age-related differences in cardiovascular reactivity to laboratory tasks. Journals Gerontol. - Ser. B Psychol. Sci. Soc. Sci. 65 B, 154-162 (2010).

53. Hines, E. A. \& Brown, G. E. The cold pressor test for measuring the reactibility of the blood pressure: Data concerning 571 normal and hypertensive subjects. Am. Heart J. 11,1-9 (1936).

54. Hermans, E. J., Henckens, M. J. A. G., Joëls, M. \& Fernández, G. Dynamic adaptation of large-scale brain networks in response to acute stressors. Trends Neurosci. 37, 304-314 (2014). 
55. van Oort, J. et al. How the brain connects in response to acute stress: A review at the human brain systems level. Neurosci. Biobehav. Rev. 83, 281-297 (2017).

56. Smeets, T. Autonomic and hypothalamic-pituitary-adrenal stress resilience: Impact of cardiac vagal tone. Biol. Psychol. 84, 290-295 (2010).

57. Castaldo, R. et al. Acute mental stress assessment via short term HRV analysis in healthy adults: A systematic review with meta-analysis. Biomed. Signal Process. Control 18, 370-377 (2015).

58. Brummett, B. H., Boyle, S. H., Kuhn, C. M., Siegler, I. C. \& Williams, R. B. Positive affect is associated with cardiovascular reactivity, norepinephrine level, and morning rise in salivary cortisol.

Psychophysiology 46, 862-869 (2009).

59. Foley, P. \& Kirschbaum, C. Human hypothalamus-pituitary-adrenal axis responses to acute psychosocial stress in laboratory settings. Neurosci. Biobehav. Rev. 35, 91-96 (2010).

60. Herman, J. P. et al. in Compr. Physiol. 603-621 (John Wiley \& Sons, Inc., 2016). doi:10.1002/cphy.c150015

61. McCubbin, J. A. Stress and endogenous opioids: Behavioral and circulatory interactions. Biol. Psychol. 35, 91-122 (1993).

62. Allen, A. J., McCubbin, J. A., Loveless, J. P. \& Helfer, S. G. Effects of estrogen and opioid blockade on blood pressure reactivity to stress in postmenopausal women. J. Behav. Med. 37, 94-101 (2014).

63. Gianaros, P. J. \& Wager, T. D. Brain-Body Pathways Linking Psychological Stress and Physical Health. Curr. Dir. Psychol. Sci. 24, 313-321 (2015).

64. Myers, B. Corticolimbic regulation of cardiovascular responses to stress. Physiol. Behav. 172, 49-59 (2017).

65. Brownley, K. A. et al. Sympathoadrenergic mechanisms in reduced hemodynamic stress responses after exercise. Med. Sci. Sports Exerc. 35, 978-986 (2003).

66. Nyhuis, T. J., Masini, C. V., Sasse, S. K., Day, H. E. W. \& Campeau, S. Physical activity, but not environmental complexity, facilitates HPA axis response habituation to repeated audiogenic stress despite neurotrophin mRNA regulation in both conditions. Brain Res. 1362, 68-77 (2010).

67. Harber, V. J. \& Sutton, J. R. Endorphins and Exercise. Sport. Med. 1, 154-171 (1984).

68. Mikkelsen, K., Stojanovska, L., Polenakovic, M., Bosevski, M. \& Apostolopoulos, V. Exercise and mental health. Maturitas 106, 48-56 (2017).

\section{Figures}




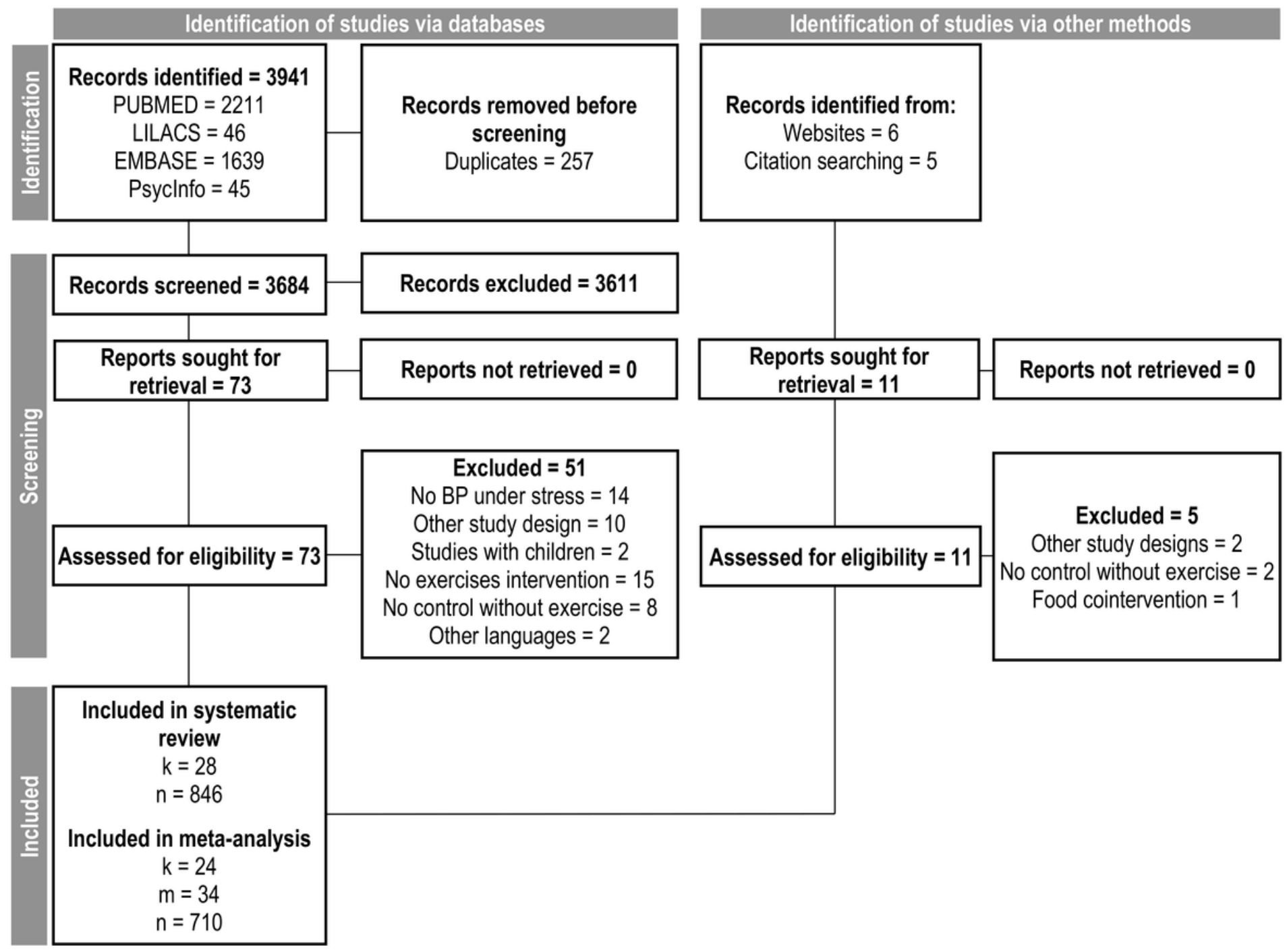

Figure 1

Flow diagram. k: number of studies, n: pooled sample size, BP: blood pressure. 
Study

\section{Aerobic}

Boone (1993)

Rejeski (1991)*

Monroe (2018)*

Ebbesen (1992)*

Ebbesen (1992)*

Rejeski (1992)

Alderman (2007)*

Hobson (1993)*

Probst (1997)

West (1998)

Rejeski (1991)*

Alderman (2007)*

Hobson (1993)*

Meireles (2020)*

Hamer (2006) ${ }^{\star}$

Someya (2012)

Meireles (2020)*

Rooks (2011)*

Hamer (2006)*

Hobson (1993)*

Szabo (1993)

Routledge (2015)

Péronnet (1989)

Rooks (2011)*

Neves (2012)

Overall effect

$I^{2}=15 \%, \tau^{2}=0.0118, \chi_{24}^{2}=28.37(p=0.24)$

\section{Resistance}

da Silva (2015)*

Heffernan (2017)

Paine (2013)

da Silva (2015)*

Overall effect

$t^{2}=24 \%, \tau^{2}=0, x_{3}^{2}=3.92(p=0.27)$

\section{Yoga}

Monroe (2018)

Benvenutti (2017)

Overall effect

$I^{2}=0 \%, \tau^{2}=0, x_{1}^{2}=0.52(p=0.47)$

Combined

Moreira (2014)

Overall effect

$I^{2}=17 \%, \tau^{2}=0.0145, \chi_{31}^{2}=37.33(p=0.20)$
Exercise vs Control

(SBP)

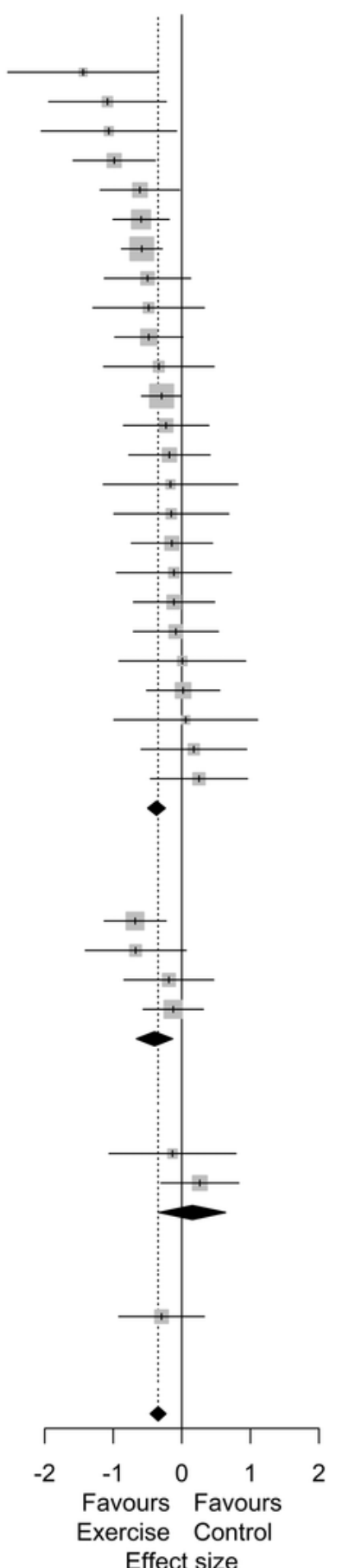

SMD

$95 \% \mathrm{Cl}$ weight

$-1.44[-2.54 ;-0.34] \quad 1.0 \%$

$-1.08[-1.94 ;-0.23] \quad 1.7 \%$

$-1.06[-2.05 ;-0.08] \quad 1.3 \%$

$-0.99[-1.58 ;-0.39] \quad 3.2 \%$

$-0.61[-1.19 ;-0.03] \quad 3.4 \%$

$-0.59[-1.00 ;-0.19] \quad 5.9 \%$

$-0.58[-0.88 ;-0.29] \quad 9.1 \%$

$-0.50[-1.13 ; 0.13] \quad 2.9 \%$

$-0.48[-1.30 ; 0.33] \quad 1.8 \%$

$-0.48[-0.98 ; 0.02] \quad 4.3 \%$

$-0.34[-1.14 ; 0.47] \quad 1.9 \%$

$-0.29[-0.59 ; 0.00] \quad 9.2 \%$

$-0.23[-0.85 ; 0.39] \quad 3.0 \%$

$-0.18[-0.77 ; 0.41] \quad 3.2 \%$

$-0.16[-1.15 ; 0.82] \quad 1.3 \%$

$-0.15[-0.99 ; 0.68] \quad 1.7 \%$

$-0.14[-0.74 ; 0.45] \quad 3.2 \%$

$-0.12[-0.95 ; 0.72] \quad 1.7 \%$

$-0.11[-0.71 ; 0.48] \quad 3.2 \%$

$-0.09[-0.71 ; 0.53] \quad 3.0 \%$

$0.01[-0.92 ; 0.93] \quad 1.4 \%$

$0.02[-0.52 ; 0.55] \quad 3.9 \%$

$0.06[-0.99 ; 1.10] \quad 1.1 \%$

$0.18[-0.60 ; 0.95] \quad 2.0 \%$

$0.25[-0.46 ; 0.96] \quad 2.4 \%$

$-0.37[-0.50 ;-0.24] \quad 76.9 \%$

$-0.68[-1.13 ;-0.23] \quad 5.1 \%$

$-0.67[-1.41 ; 0.06] \quad 2.2 \%$

$-0.19[-0.84 ; 0.47] \quad 2.7 \%$

$-0.13[-0.56 ; 0.31] \quad 5.3 \%$

$-0.40[-0.66 ;-0.13] 15.3 \%$

$-0.14[-1.06 ; 0.79] \quad 1.4 \%$

$0.26[-0.31 ; 0.83] \quad 3.5 \%$

$0.15[-0.33 ; 0.64] \quad 4.9 \%$

$-0.30[-0.92 ; 0.33] \quad 3.0 \%$

$-0.35[-0.46 ;-0.23] 100.0 \%$

\section{Figure 2}

Systolic blood pressure reactivity forest plot. SMD: standardized mean difference; SBP: systolic blood pressure; Cl: credible interval; *: studies with multiple comparisons. 
Study

Aerobic

Ebbesen (1992)*

Boone (1993)

Ebbesen (1992)*

Monroe (2018)*

West (1998)

Rejeski (1991)*

Rejeski (1991)*

Hobson (1993)*

Alderman $(2007)^{\star}$

Hamer (2006)*

Probst (1997)

Rejeski (1992)

Meireles (2020)*

Alderman (2007) ${ }^{\star}$

Hobson (1993)*

Hamer (2006)*

Szabo (1993)

Hobson (1993)*

Meireles (2020)*

Routledge (2015)

Someya (2012)

Rooks (2011)*

Santaella (2006)

Rooks (2011)*

Péronnet (1989)

Neves (2012)

Overall effect

$I^{2}=74 \%, \tau^{2}=0.2440, x_{25}^{2}=96.83(p<0.01)$

\section{Resistance}

Heffernan (2017)

da Silva (2015)*

da Silva (2015)*

Paine (2013)

Overall effect

$l^{2}=57 \%, \tau^{2}=0.0558, x_{3}^{2}=7.01(p=0.07)$

Yoga

Monroe (2018)*

Benvenutti (2017)

Overall effect

$t^{2}=0 \%, \tau^{2}=0, x_{1}^{2}=0.2(p=0.66)$

\section{Combined}

Moreira (2014)

Overall effect

$I^{2}=70 \%, \tau^{2}=0.1979, x_{32}^{2}=106.09(p<0.01)$

\section{Exercise vs Control}

(DBP)

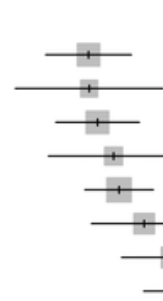

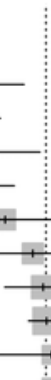

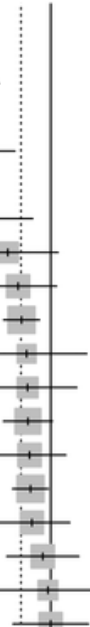

$2.07[-2.78 ;-1.37] \quad 3.0 \%$

$-2.06[-3.28 ;-0.85] \quad 1.7 \%$

$-1.93[-2.61 ;-1.24] \quad 3.0 \%$

$-1.66[-2.73 ;-0.59] \quad 1.9 \%$

$-1.57[-2.13 ;-1.01] \quad 3.5 \%$

$-1.16[-2.03 ;-0.30] \quad 2.5 \%$

$-0.71[-1.53 ; 0.12] \quad 2.6 \%$

$-0.54[-1.17 ; 0.09] \quad 3.2 \%$

$-0.48[-0.78 ;-0.18] \quad 4.4 \%$

$-0.40[-1.39 ; 0.59] \quad 2.1 \%$

$-0.38[-1.19 ; 0.43] \quad 2.6 \%$

$-0.38[-0.78 ; 0.03] \quad 4.0 \%$

$-0.35[-0.95 ; 0.25] \quad 3.3 \%$

$-0.33[-0.63 ;-0.04] \quad 4.4 \%$

$-0.31[-0.93 ; 0.31] \quad 3.2 \%$

$-0.13[-0.72 ; 0.46] \quad 3.3 \%$

$-0.05[-0.97 ; 0.88] \quad 2.3 \%$

$-0.00[-0.62 ; 0.62] \quad 3.2 \%$

$0.00[-0.59 ; 0.59] \quad 3.3 \%$

$0.02[-0.52 ; 0.55] \quad 3.6 \%$

$0.04[-0.80 ; 0.88] \quad 2.5 \%$

$0.07[-0.70 ; 0.84] \quad 2.7 \%$

$0.10[-0.41 ; 0.61] \quad 3.7 \%$

$0.26[-0.58 ; 1.10] \quad 2.5 \%$

$0.32[-0.73 ; 1.38] \quad 2.0 \%$

$0.42[-0.29 ; 1.13] \quad 2.9 \%$

$-0.48[-0.71 ;-0.24] \quad 77.6 \%$

$-0.90[-1.66 ;-0.15] \quad 2.8 \%$

$-0.80[-1.25 ;-0.34] \quad 3.8 \%$

$-0.43[-0.87 ; 0.02] \quad 3.9 \%$

$0.18[-0.47 ; 0.84] \quad 3.1 \%$

$-0.50[-0.86 ;-0.14] 13.6 \%$

$-0.96[-1.93 ; 0.02] \quad 2.2 \%$

$-0.70[-1.29 ;-0.12] \quad 3.4 \%$

$-0.77[-1.27 ;-0.27] \quad 5.5 \%$

$-0.30[-0.93 ; 0.32] \quad 3.2 \%$

$-0.49[-0.68 ;-0.30] 100.0 \%$

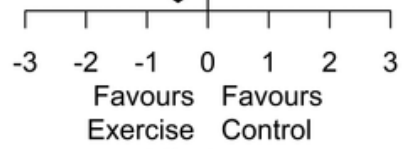

Exercise Control

Effect size

\section{Figure 3}

Diastolic blood pressure reactivity forest plot. SMD: standardized mean difference; DBP: diastolic blood pressure; Cl: credible interval; *: studies with multiple comparisons. 
Study

Aerobic

Boone (1993)

West (1998)

Bartholomew (2000)

Rejeski (1991)*

Rejeski (1992)

Rejeski (1991)*

Hobson (1993)*

Probst (1997)

Hobson (1993)*

Hobson (1993)*

Someya (2012)

Neves (2012)

Rooks (2011)*

Rooks (2011)*

Overall effect

$I^{2}=51 \%, \tau^{2}=0.1085, \chi_{13}^{2}=26.47(p=0.01)$

\section{Resistance}

da Silva (2015)*

da Silva (2015)*

Overall effect

$I^{2}=0 \%, \tau^{2}=0, \chi_{1}^{2}=0.72(p=0.40)$

Overall effect

$I^{2}=46 \%, \tau^{2}=0.0776, \chi_{15}^{2}=27.97(p=0.02)$
Exercise vs Control

(MBP)

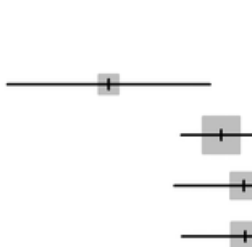

$-2.60[-3.94 ;-1.27] \quad 2.3 \%$

$-1.12[-1.65 ;-0.60] \quad 8.2 \%$

$-0.83[-1.74 ; 0.09] \quad 4.2 \%$

$-0.81[-1.64 ; 0.02] \quad 4.8 \%$

$-0.72[-1.13 ;-0.31] \quad 10.1 \%$

$-0.59[-1.41 ; 0.23] \quad 4.9 \%$

$-0.54[-1.17 ; 0.09] \quad 6.8 \%$

$-0.53[-1.34 ; 0.29] \quad 4.9 \%$

$-0.27[-0.89 ; 0.36] \quad 6.9 \%$

$-0.19[-0.82 ; 0.43] \quad 6.9 \%$

$-0.10[-0.94 ; 0.73] \quad 4.7 \%$

$0.07[-0.64 ; 0.77] \quad 6.0 \%$

$0.10[-0.73 ; 0.94] \quad 4.7 \%$

$0.13[-0.64 ; 0.90] \quad 5.3 \%$

$-0.51[-0.77 ;-0.25] \quad 80.8 \%$

$-0.50[-0.94 ;-0.05] \quad 9.5 \%$ $-0.23[-0.67 ; 0.21] \quad 9.6 \%$

$-0.36[-0.68 ;-0.05] \quad 19.2 \%$

$-0.48[-0.70 ;-0.26] 100.0 \%$

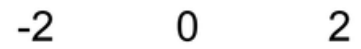

Favours Favours

Exercise Control

Effect size

Figure 4

Mean blood pressure reactivity forest plot. SMD: standardized mean difference; MBP: mean blood pressure; Cl: credible interval; *: studies with multiple comparisons. 


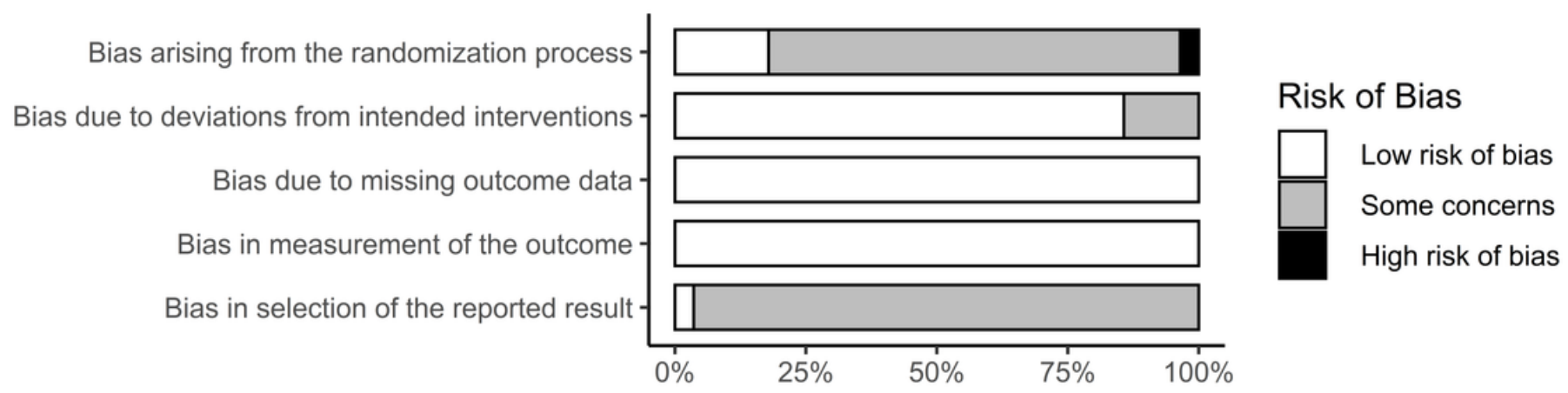

\section{Figure 5}

Risk of bias summary ( $k=28)$.
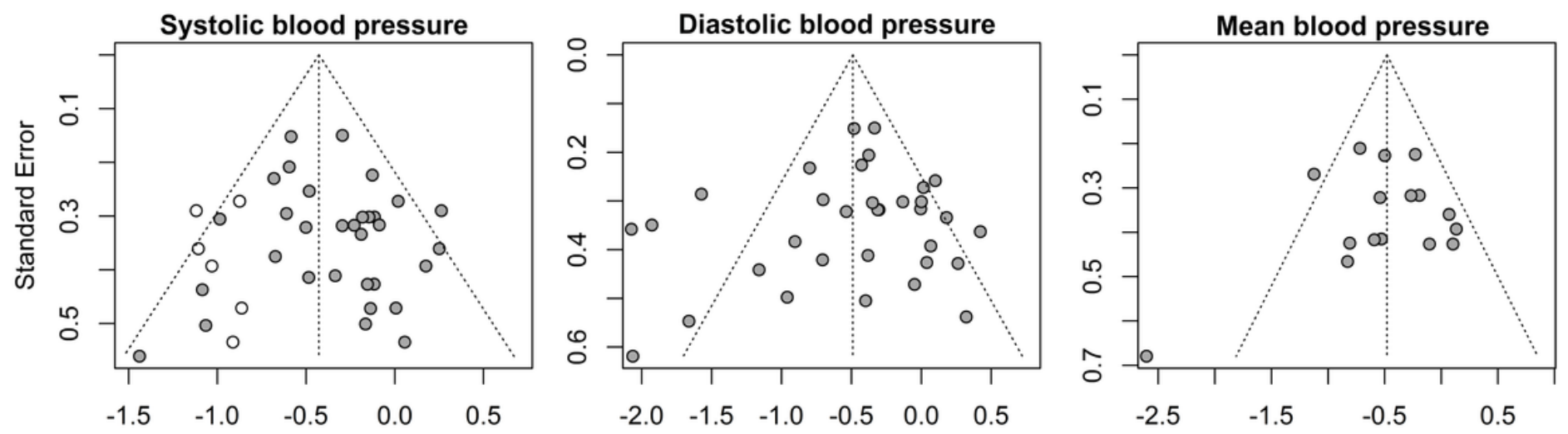

Standardised Mean Difference

\section{Figure 6}

Publication bias representation by trim and fill funnel plots. White circles represent possible omitted studies.

\section{Supplementary Files}

This is a list of supplementary files associated with this preprint. Click to download.

- SuplementV2.pdf 